\title{
ECOMOSAICO FLORESTAL: COMPOSIÇÃO, ÍNDICES DE INFORMAÇÃO E ABDUÇÃO ${ }^{1}$
}

\author{
José Armando Pinto Casquilho²
}

\begin{abstract}
RESUMO - Neste trabalho, apresenta-se um conjunto de índices de informação sobre a composição do mosaico de paisagem, que se estende ao conceito de ecomosaico. Esses índices são generalizações sucessivas da função de Shannon, utilizada para medir a diversidade da paisagem e também interpretada como entropia estatística, ou valor médio de informação de um sistema canônico, um parâmetro macroscópico de um sistema estocástico; ainda podem ser considerados valores médios, ou esperados, de preferências, aparentadas com funções de utilidade usadas num sentido amplo do conceito. Os índices de informação discutidos são exemplificados através de um cenário hipotético de planejamento na região de Nisa, em Portugal, caracterizado por indicadores econômicos do valor de habitats florestais característicos da região. Os índices são também utilizados para referenciar uma reflexão que se processa à volta de três balizas respeitantes ao conceito de informação: as teorias da situação, da relevância e da decisão. No seu âmbito mais lato, trata-se de um exercício de abdução, operacionalizando uma estratégia racional que pode servir de base à tomada de decisões sobre o planejamento do território ou, ainda, para investigar a dinâmica de alterações na composição da paisagem. No caso exemplificado, os índices apontaram que o montado de sobro e o pinhal manso são de longe os habitats florestais mais promissores na região, num cenário sem restrições biofísicas.
\end{abstract}

Palavras-chave: Valores de informação, Valores característicos e Utilidade esperada.

\section{FOREST ECOMOSAIC: COMPOSITION, INFORMATION INDICES, ABDUCTION}

\begin{abstract}
This work presents a set of indices of information on the composition of landscape mosaics which extends to the concept of ecomosaic. These indices are successive generalizations of the Shannon function, used to measure landscape diversity, and interpreted as statistical entropy, or average value of information of a canonical system, a macroscopic parameter of a stochastic system; the formulas can also be considered to represent average or expected values of preferences, related with utility functions used in a broad sense of the concept. The information indices discussed in this paper are exemplified by a hypothetical scenario planning in the region of Nisa, Portugal, characterized by economic indicators of the value of forest habitats. The indices are also used to anchor a reflection that goes around three beacons relating to the concept of information: the Theories of Situation, Relevance, and Decision. The whole exercise may be seen as an abduction procedure, running a rational strategy that can serve as a basis for decision-making on the planning of the territory or also, it serves to investigate dynamic changes in the composition of the landscape mosaic. In the case exemplified the indices show that the cork oak and the umbrella pine forests are, by far, the most promising habitats in the region, under a scenario with no biophysical constraints.
\end{abstract}

Keywords: Information values, Characteristic values and Expected utility.

\footnotetext{
${ }^{1}$ Recebido em 19.04.2010 e aceito para publicação em 29.03.2012

${ }^{2}$ Centro de Ecologia Aplicada Baeta Neves, Lisboa, Portugal. Faculdade de Agricultura da Universidade Nacional Timor Lorosa'e, Díli, Timor-Leste. E-mail: <josecasquilho@gmail.com>.
} 


\section{INTRODUÇÃO}

O mosaico de paisagem é uma designação que constitui uma metonímia. Feito de tesselas e inspirado pelas musas, são as raízes da palavra mosaico a que se junta a paisagem, agregado de pagus, demarcação rural do Império Romano. A etimologia remete ainda para a raíz indo-europeia de pacto e de paz ligada a sentido moral (LEPECKI, 2001). Como instrumento analítico, serve para qualificar e quantificar componentes e elementos da paisagem analisada enquanto objecto geográfico e ecológico.

A Ecologia da Paisagem surgiu pela mão do geógrafo Carl Troll, que estudava fotografia aérea nos anos trintas do século passado, desenvolvendo-se num progressivo casamento entre geografia e biologia (ZONNEVELD, 1990), mas só se afirmou como ciência emergente no final do século XX. Uma definição recente de Ecologia da Paisagem é “a ciência e a arte de estudar e influenciar a relação entre padrões espaciais e processos ecológicos através de diferentes níveis hierárquicos da organização biológica e diferentes escalas no espaço e no tempo" (WU; HOBBS, 2007).

Ecomosaico é um termo cunhado por Forman (1979), referido numa escala em que tende a compreender regiões compostas por paisagens, sendo estas compostas por ecossistemas onde a geomorfologia e a cultura em interacção produzem os mosaicos (FORMAN, 1995). Para além da metonímia expressa em mosaico de paisagem, o termo ecomosaico remete mais para a ordem da metáfora - a composição de paisagens expressa-se num padrão que reflecte tanto a ecologia como a economia da região entrelaçadas: natureza e cultura.

Nagendra e Gadgil (1999) consideraram ecomosaicos unidades de observação na escala de $10^{8}-10^{10} \mathrm{~m}^{2}$, que se colocam entre as ecorregiões e os ecótopos. O termo ecomosaico pode ser utilizado ainda como referência, visando a uma caracterização sumária de mosaicos de paisagens através de classes, índices e medidas de natureza ecológica ou económica.

Gomes (1985) situava os termos da sustentabilidade de mosaicos florestais centrados no conceito de uso múltiplo das florestas - antes delineado por Alves (1963) -, enunciando que tal se concretiza consorciando funções diversas nas mesmas áreas e compartimentando áreas por funções distintas, ou grupos de funções, num mosaico que garanta um fluxo sustentável e graduado de bens e de benefícios indirectos.

Entre as características básicas de um mosaico de paisagem, destacam-se a composição e a configuração, a primeira relativa aos habitats presentes e números de manchas de diferentes tipos e proporções da área total e, a segunda, relativa ao arranjo espacial dos elementos: fragmentação e conectividade, entre outros. A composição de um mosaico de paisagem, ou de um ecomosaico, é uma abstração que se pode expressar em configurações variadas concretas.

As manchas de habitats foram definidas como componentes estruturais do mosaico de paisagem (FORMAN; GODRON, 1981) e afectam processos, incluindo a ocorrência de fogos (MOREIRA et al., 2001). Sobre a composição incidem medidas introduzidas por analogia com a ecologia das comunidades: a riqueza em espécies é substituída pela riqueza em habitats, e a diversidade da comunidade é substituída pela diversidade da paisagem, o mais das vezes avaliada pela fórmula de Shannon (1948), embora também se conheçam métodos baseados na fórmula de Simpson (1949). A fórmula de Shannon é considerada o único funcional significativo para medir a informação relacionada com a incerteza em teoria das probabilidades (KLIR, 2006). Kornreich (2008) esclareceu que se trata de um parâmetro macroscópico de um sistema estocástico.

Hansen (1993) referiu que no quadro da teoria dos sistemas dinâmicos se pode tratar um problema equivalentemente do ponto de vista do mínimo de uma função potencial ou do máximo de uma distribuição de probabilidades que ocorrem entre si numa relação simétrica. Também é tempo de fazer um alerta sobre reificação: trata-se de uma falácia filosófica em que se toma uma abstração como se fora um objecto real, e esse risco existe frequentemente em Ecologia (BOWMAN, 2007).

Não obstante, recolocando o quadro teórico subjacente: existe a Teoria da Decisão, que se sustenta na análise da utilidade de uma opção (WEIRICH, 2001) e se desenvolve afirmando que os decisores se regem procurando maximizar utilidades esperadas (GILBOA, 2009) definidas dentro de um quadro de racionalidade estocástica subjectiva, enquanto a Teoria da Situação afirma que há mais informação disponível do que aquela que se pode representar (PARIKH; CLARK, 2007). Ainda, 
a Teoria da Relevância, em que a relevância é definida em termos da contrapartida entre o esforço necessário para processar algum input tal como uma declaração verbal ou matemática e o ganho informacional de empreender esse processamento inferencial (CANN et al., 2009). Outrossim, do lado da Ecologia, Allen et al. (2010b) recordaram que a perda de resiliência ecológica pode acontecer por via da implementação de estratégias de controle ótimo numa única variável alvo.

O trabalho que aqui se apresenta consiste na discussão de índices que resultam de generalização da fórmula de Shannon, na demanda de internalizar na análise da composição um conjunto de valores intrínsecos ou característicos dos habitats presentes num mosaico de paisagem, real ou imaginário, e, ainda, valores de informação relacionados com a sua expressão no contexto. Stratton-Lake (2004) referiu que o valor contributivo, diferentemente do valor intrínseco, é uma forma relacional de valor que uma parte confere ao todo a que pertence condicionada pela presença das restantes.

No caso da fórmula de Shannon, o valor de informação é um número real positivo que traduz uma medida logarítmica da raridade expressa pelo inverso multiplicativo da probabilidade. Dá-se, assim, por aberta uma discussão em que as noções associadas aos índices de valor informativo médio, de valor contributivo médio e de valor indicativo médio, que se apresentam a seguir, podem ajudar a situar decisões ou a avaliar diagnósticos sobre a composição do mosaico de paisagem ou ecomosaico florestal.

\section{MATERIAL E MÉTODOS}

A fórmula de Shannon é também designada entropia estatística ou valor médio de informação; representamola com logaritmos naturais e a notação $H=-\sum_{i=1}^{n} x_{i} \log x_{i}$, em que as variáveis $\left\{x_{i}\right\}_{i=1, \ldots, n}$ denotam probabilidades de acontecimentos, frequências relativas, ou proporções - em qualquer caso medidas de extensão relativa -, números reais que verificam as condições: $x_{i} \geq 0$ e $\sum_{i=1}^{n} x_{i}=1$, definindo um simplex $n-1$ dimensional.

Tem-se $0 \leq H \leq \log n$ acontecendo o valor mínimo quando a incerteza, ou diversidade, é nula: o mosaico está completamente preenchido por um, e apenas um, dos tipos de habitats considerados, e está-se num vértice do simplex; o valor máximo $H^{*}=\log n$ ocorre na chamada situação de indiferença: os acontecimentos geradores são equiprováveis ou, na linguagem da diversidade da paisagem: os diferentes habitats ocupam áreas iguais com $x_{i}^{*}=1 / n$ para $i=1, \ldots, n$.

A quantidade $y_{i}=-\log x_{i}$ é designada valor de informação do habitat $h_{i}$ e traduz-se na ideia de que um número real positivo expressa a medida de que o valor de informação de algo que se tem por certo é zero, enquanto um acontecimento muito improvável acrescenta muita informação se realizado; quanto mais improvável um acontecimento, ou quanto mais raro é o estado do sistema associado, maior é o seu valor de informação, expresso como número real positivo - a ideia de fundo é de que um grande desvio em relação a uma situação estatisticamente genérica engendra uma saliência perceptiva e uma significação própria (PETITOT, 2004), e, quanto menos uma situação é provável, mais ela é intrinsecamente significativa.

As propriedades da generalização da função $H$ que se apresenta a seguir estão demonstradas em Casquilho et al. (1997) e faladas em Ricotta (2002), no âmbito das medidas de diversidade e biodiversidade em Ecologia ou, mais recentemente, na sua proximidade ao conceito de diversidade filogenética (ALLEN et al., 2009); comporta a introdução de valores intrínsecos ou característicos dos habitats, expressos por números reais positivos, formando um conjunto $\mathrm{W}=\left\{w_{1}, \ldots, w_{n}\right\}$, e podem ser estruturados em ponto ou vetor $w$.

\subsection{Valor informativo médio}

A extensão imediata da função $H$ é $H_{w}=-\sum_{i=1}^{n} w_{i} x_{i} \log x_{i}$, uma medida de diversidade ponderada por valores característicos que se pode interpretar ainda como o valor médio, ou valor esperado, de uma variável aleatória discreta que toma valores $z_{i}=-w_{i} \log x_{i}$ com probabilidades $P\left[Z=z_{i}\right]=x_{i}$ para $i=1, \ldots, n$; os números reais $z_{i}$ são formados pelo produto do valor característico e do valor de informação e designa-se como valor informativo relativo ao habitat $h_{i}$. A função $H_{w}$ pode, então, interpretar-se como um índice de valor informativo médio no sentido anteriormente especificado, ou uma medida de diversidade ponderada por valores característicos ou intrínsecos dos habitats. Mostra-se que $H_{w}$ é uma função contínua definida no simplex $n$ - 1 dimensional e alcança valor mínimo e valor máximo para cada concretização de $\mathrm{W}$, podendo se escrever:

Revista Árvore, Viçosa-MG, v.36, n.2, p.321-329, 2012 
$0 \leq H_{w} \leq H_{w}^{*}$, em que $H_{w}^{*}$ designa o valor máximo que ocorre no único ponto de coordenadas óptimas $x^{*}=\left(x_{1}^{*}, \ldots, x_{n}^{*}\right)$; mostra-se, ainda, que $0<x_{i}^{*}<e^{-1}$ para $i=1, \ldots, n$ e que as coordenadas da solução óptima podem ser obtidas por um procedimento que se apresenta mais à frente.

\subsection{Valor contributivo médio}

Outra extensão da função $H$ pode ser construída como se sucede: seja $W_{T}=\sum_{i=1}^{n} w_{i} x_{i}$ a média dos valores do conjunto W. Então, define-se valor contributivo médio como $K_{w}=W_{T}+H_{w}=\sum_{i=1}^{n} w_{i} x_{i}\left(1-\log x_{i}\right)$. A designação de valor contributivo refere-se aos valores $c_{i}=w_{i}\left(1-\log x_{i}\right)$, o produto do valor característico $w_{i}$ pelo factor de informação $f_{i}=1-\log x_{i}=1+y_{i}$; o factor de informação é o valor de informação atrás referido adicionado à unidade. Sendo $C$ uma variável aleatória discreta que toma valores com probabilidade $P[C=$ $\left.C_{i}\right]=x_{i}$ para $i=1, \ldots, n$, tem-se $K_{w}=E[C]$, um valor médio ou valor esperado. Por que valor contributivo? Porque contribui na soma geral com o seu valor característico ou intrínseco $w_{i}$ e, ainda, com o factor de informação relativo ao contexto. Da mesma forma que para a função $H_{w}$, também se conclui que a função $K_{w}$ é uma função contínua definida no simplex e alcança valor mínimo e valor máximo, em que $K^{*}$ designa o valor máximo que ocorre no ponto de coordenadas óptimas; mostra-se também que $0<x_{i}^{*}<1$ para $i=1, \ldots, n$ e as coordenadas da solução óptima podem ser obtidas por um procedimento descrito no item 2.4.

\subsection{Valor indicativo médio}

A última extensão da função $H$ que se apresenta arruma as duas anteriores numa só fórmula geral com um parâmetro $\alpha, \alpha \in[0,1]$, podendo escrever-se: $I_{w}(\alpha ; x)=\sum_{i=1}^{n} w_{i} x_{i}\left(\alpha-\log x_{i}\right)$, verificando-se que $I_{w}(0 ; x)=H_{w}$ e $I_{w}(1 ; x)=K_{w}$. Na família $I_{w}(\alpha ; x)$, o representante médio obtém-se fazendo $\alpha=0.5$, denotando $I_{w}(0.5 ; x)=I_{w}$. Esse índice que se designa por valor indicativo médio apresenta as mesmas propriedades geométricas dos casos descritos anteriormente: é uma função côncava e contínua e tem valor mínimo e valor máximo definidos de maneira única no simplex para cada concretização de W, como apresentado nos tópicos subsequentes.

\subsection{Soluções óptimas}

Para qualquer dos casos referidos, tem-se a solução óptima definida pelas fórmulas [1] que envolvem a resolução numérica de uma equação para obter uma das coordenadas do maximizante, sendo as outras coordenadas explicitáveis a partir dela:

$$
\begin{aligned}
& x_{i}^{*}: \sum_{j=1}^{n} x_{i}^{\frac{w_{i}}{w_{j}}} e^{(\alpha-1) \frac{w_{j}-w_{i}}{w_{j}}}=1 ; x_{j}^{*}=x_{i}^{* \frac{w_{i}}{w_{j}}} e^{(\alpha-1) \frac{w_{j}-w_{i}}{w_{j}}} \\
& \text { para } j=1, \ldots, n(j \neq i)
\end{aligned}
$$

Fixa-se $\alpha=0$ para obter a solução óptima do índice $H_{w}$ e $\alpha=1$ para obter a solução correspondente para $K_{w}$. A família $I_{w}(\alpha)$ faz, assim, um compromisso entre os índices $H_{w}$ e $K_{w}$, ponderando o grau de participação de $W_{T}$; os índices têm em comum o fato de serem todos valores médios de variáveis aleatórias discretas, que em qualquer caso internalizam valores característicos definidos pelo conjunto $\mathrm{W}$, caracterizando um cenário do mundo, e valores de informação.

\section{RESULTADOS}

Admita-se que há pela frente um exercício de planejamento ou de diagnóstico simplificado. Existe uma área vasta, sem restrições, e dispõe-se de um conjunto de habitats florestais característicos da região para poder replicar indistintamente. Os economistas do ambiente consideram que as mudanças na paisagem reflectem a atribuição de valores económicos, mesmo que implícitos (SANTOS, 2001), e a ideia de fundo é que, dentro de certa racionalidade normativa, os agentes de decisão buscam a maximização de uma utilidade esperada, estendida para o conceito de utilidade subjectiva esperada (GILBOA, 2009), em que ainda se pode considerar o utilitarismo ponderado como escolha racional por parte de um grupo (WEIRICH, 2001).

Esse conceito de utilidade esperada pode ser centrado no valor económico, mas também pode estar vinculado à preservação da biodiversidade, reflectindo uma utilidade multidimensional. O conceito de função de utilidade vem da teoria dos jogos, e a forma normal é a de um valor médio de funções de preferência afectadas da respectiva distribuição de probabilidades (BLACKWELL; GIRSHICK, 1979).

Nesse caso, pode-se escrever a família de funções com 1-parâmetro $\alpha$ : $\mathrm{U}_{a}()=.I_{w}(\alpha ; x)$, em que as funções 
de preferência são descritas como $p_{i}=w_{i}\left(\alpha-\log x_{i}\right)$ com $\alpha \in[0,1]$, que se desdobra nos três casos típicos anteriormente referidos: valor informativo, valor contributivo e valor indicativo.

Pode-se, então, interpretar $I_{w}(\alpha ; x)=U_{\alpha}(y \mid w)$, em que $\sum_{i=1}^{n} x_{i}=1 \Rightarrow \sum_{i=1}^{n} e^{-y i}=1$; sendo $U_{a}$ (.) um funcional de utilidade esperada de parâmetro $\alpha$ que comporta valores característicos e valores de informação e onde $y \mid w$ se lê: o vetor $y$ dado o vetor $w$, constituído pelos valores do conjunto $\mathrm{W}$ que caracterizam um cenário.

O caso que se apresenta em seguida visa ilustrar o desempenho dos índices $H_{w}, K_{w}$ e $I_{w}$, e utiliza informação económica: valores unitários expressos em euros por hectare. Na região de Nisa, junto ao Tejo e à Espanha, existem estimativas recentes do valor económico de 1 ha de diversos tipos de povoamentos florestais calculados a partir de valores de mercado na perspectiva da rendibilidade média e também do custo de reconstituição em caso de incêndio (PMDFCI, 2007). Do total de tipos de habitats florestais listados, extraiu-se um conjunto de cinco com relevância na região, como: povoamentos ou montados de sobreiro [sb] (Quercus suber L.); povoamentos ou montados de azinheira [az] (Q. Rotundifolia Lam.); eucaliptais [eg] (Eucalyptus globulus Labill.); e pinhais, manso [pm] (Pinus pinea L.) e bravo [pb] ( $P$. pinaster Aiton). Os valores económicos florestais expressos em euros por hectare, bem como as soluções óptimas calculadas para cada índice, são apresentados na Tabela 1.

Pode-se dizer, em face dos valores apresentados na Tabela 1 , que a gama de soluções equilibradas de composição do mosaico parece ser algo que se reparte, como: sobro, 30\%-50\%; azinho, 2\%-13\%; eucaliptal 4\%-16\%; pinhal manso, 29\%-42\%; e pinhal bravo, $1 \%$ $10 \%$, tendo como valores centrais, ou de referência, o conjunto: $\mathrm{S}=$ s sb_41\%, az_7\%, eg_11\%, pm_37\%, pb_4\%\}. Em relação aos valores máximos apresentados, há que salientar que, enquanto os valores característicos são expressos em $€ / h a$, os valores dos índices podem ser lidos em $€ /$ ha, em que ui designa unidade de informação, aparentada a bit ( bit).

\section{DISCUSSÃO}

Os mosaicos podem ser entendidos como estados de um sistema dinâmico que coexistem numa área vasta, em proporções variáveis e evolutivas; expressam uma metamorfose da paisagem em estádios e representam uma variedade de equilíbrios de um sistema estocástico associado. A paisagem pode evoluir rapidamente e, por efeito da expansão das monoculturas extensivas a sua diversidade pode baixar drasticamente, diminuindo o valor médio de informação que lhe está associado. Pelo contrário, também se pode fazer restauração de habitats e recuperar a diversidade, incluindo biodiversidade, num arranjo espacial ecológico onde a própria mata regenera, a partir de plantações de eucalipto em antigas pastagens (AMARAL et al., 2009).

Da composição, e em particular das proporções de diferentes tipos de habitats, pode depender a dominância, ou a falta de dominância, de recursos críticos (LI; WU, 2007). Já nos anos de 1980 se estabelecia que o número de espécies num habitat é determinado

Tabela 1 - Valores característicos $\left(w_{i}\right)$ expressos em $€ /$ ha para os habitats florestais: montado de sobro-sb, de azinho-az, eucaliptal-eg, pinhal manso-pm, pinhal bravo-pb e soluções ótimas dos índices de valor informativo médio $H_{w}$, valor contributivo médio $K_{w}$ e valor indicativo médio $I_{w}$, expressos nos valores dos maximizantes $x_{i}^{*}$ e nos valores máximos dos índices, respectivamente $H_{w}^{*}, K_{w}^{*}$ e $H_{w}^{*}$

Table 1 - Characteristic values $w_{i}$ expressed in $€ /$ ha of forest habitats: cork oak forest- sb, holm oak groves - az, eucalyptus trees stands - eg, umbrella pine trees-pm, maritime pine stands-pb and optimal solutions of indices: mean informative value $H_{w}$, mean contributive value $K_{w}$ and mean indicative value $I_{w}$, expressed in the values of maximum point coordinates $x_{i}^{*}$ and maximum values of the indices, respectively denoted $H_{w}^{*}, K_{w}^{*}$ e $H_{w}^{*}$

\begin{tabular}{|c|c|c|c|c|c|c|}
\hline & $\mathrm{sb}$ & $\mathrm{az}$ & eg & $\mathrm{pm}$ & $\mathrm{pb}$ & máximo \\
\hline$w_{\mathrm{i}}$ & 618 & 112 & 136 & 494 & 91 & \\
\hline$H_{w}: x_{i}^{*}$ & 0.3064 & 0.1342 & 0.1603 & 0.2927 & 0.1063 & $H_{w}^{*} 493$ \\
\hline$K_{w}: x_{i}^{*}$ & 0.5022 & 0.0224 & 0.0437 & 0.4224 & 0.0093 & $K_{w}^{*} 954$ \\
\hline$I_{w}: x_{i}^{*}$ & 0.4108 & 0.0706 & 0.1032 & 0.3725 & 0.0430 & $I_{w}^{*} 709$ \\
\hline
\end{tabular}


por factores históricos e ecológicos (GILLER, 1984) e o número de nichos potenciais determinaria quantas espécies poderiam coexistir, mantendo-se a coexistência como função, quer do número de recursos discretos presentes, quer do máximo tolerável de sobreposição de nichos, ou de ambos.

Solé e Bascompte (2006) enfatizaram como a organização espacial é um aspecto-chave para compreender a estabilidade das populações e a diversidade das espécies. A paisagem pode evoluir rapidamente; por exemplo, na Serra do Açor, em Portugal, o mosaico possui elevada dinâmica de alteração da cobertura vegetal caracterizada pela diminuição da área agrícola e da área de pinheiro-bravo e pelo aumento da área de incultos e de folhosas diversas num período de 30 anos (GASPAR; FIDALGO, 2002). Cemin et al. (2009) apresentaram uma análise de fragmentos em número e proporções para três tipos de floresta na sub-bacia do Arroio Jacaré, localizada no Vale do Taquari, RS, afirmando que a progressiva fragmentação tem contribuído para a perda de biodiversidade na região.

A paisagem é um conjunto de valores ordenados numa visão (CAUQUELIN, 2008) que parece ser um enunciado potente procurando abranger a paisagem como signo, como espaço de significação e mensagem ou como um híbrido de espaço construído e natural. O valor de uma paisagem só se faz presente numa cultura, pois a valorização é cultural. Falar em maximização de qualquer utilidade esperada é supor que se conseguiu reduzir toda uma multidimensionalidade a uma função real. Recorde-se que Lepecki (2001) acentuou que o processo de nomeação e numeração não é de modo algum inocente, pois se trata de hierarquizar.

O nome que se prefere dar a $H_{w}, K_{w}$ e $I_{w}$ é o de índices de informação relativos à composição do mosaico, que se podem tomar como relacionados com funções de utilidade que estabelecem regras de preferência de estratégias que visam alcançar soluções óptimas. A Tabela 2 organiza os diferentes índices e suas soluções óptimas expressas nas mesmas medidas: o valor económico florestal médio $w_{T}=\sum_{i=1}^{n} w_{i} x_{i}$ de 1 ha e a diversidade da paisagem calculada pela fórmula $H$ de Shannon. A diversidade paisagística é máxima para uma repartição equitativa das áreas pelos cinco diferentes habitats e corresponde a determinado valor econômico calculado; pelo meio estão representados os valores que resultam dos maximizantes $x^{*}=\left(x_{1}^{*}, \ldots, x_{n}^{*}\right)$ dos diferentes índices aplicados nas duas medidas que constituem as colunas da Tabela 2.

Os resultados indicaram que se quiser um compromisso equilibrado das duas medidas em jogo - valor económico por um lado e diversidade ou valor de informação médio por outro -, o índice $I_{w}$ é o que fornece simultaneamente soluções acima dos valores centrais dos intervalos de variação do conjunto dos índices nas duas categorias de medida utilizadas.

No final do século passado, Forman (1990) deixou como hipótese provocativa que existe uma configuração óptima de ecossistemas e usos da terra de forma a maximizar a integridade ecológica e a sustentabilidade do ambiente. Neste trabalho, não se foi tão longe e não se falou sequer de configuração. Em certo sentido, a montante do problema da configuração está o da composição, e em relação a esse admite-se que o conjunto dos índices agora apresentados ajude a prospectar soluções.

Hof e Flather (2007) trataram o problema da optimização da paisagem como capturar relações espaciais entre diferentes áreas de solo no processo de maximizar ou minimizar uma função objetivo sujeita a restrições de recursos, mas, ainda assim, referiram que se trata de um processo interativo.

Holling (1973) colocou que o mundo natural consiste num mosaico de elementos espaciais com características biológicas, físicas e químicas, distintas, que estão ligados

Tabela 2 - Cálculo da medida económica do valor médio de 1 ha $\left(w_{T}\right)$ e da diversidade paisagística $(H)$ com os valores dos maximizantes dos diferentes índices relatados na Tabela 1, reportados aos valores máximos dos índices $H_{w}^{*}, K_{w}^{*}$ e $I_{w}^{*}$; o valor máximo do índice de Shannon, denotado $H^{*}$, serve de referência

Table 2 - Calculation of average economic value of a hectare $\left(w_{T}\right)$ and landscape diversity $(H)$ with the values obtained with maximizing points of different indexes reported in Table 1 , designated $H_{w}^{*}, K_{w}^{*}$ e $I_{w}^{*}$, and, the maximum value of Shannon index, named $H^{*}$, guides as a reference

\begin{tabular}{|c|c|c|c|}
\hline Máximos / & Medidas & $w_{T}(\in / h a)$ & $H(\sim$ bits $)$ \\
\hline$H^{*}$ & & 290 & 1.6089 \\
\hline$H_{w}^{*}$ & & 381 & 1.5234 \\
\hline$K_{w}^{*}$ & & 528 & 0.9752 \\
\hline$I_{w}^{*}$ & & 464 & 1.2901 \\
\hline
\end{tabular}


por mecanismos de transportes físico e biológico. Determinar as escalas presentes em sistemas é importante para a teoria da resiliência e da panarquia (ALLEN et al., 2010a), e esta incide na possibilidade da existência de equilíbrios múltiplos na dinâmica dos ecossistemas e no estudo dos factores que afetam as velocidades, ou taxas, de eventual recuperação do sistema para um equilíbrio anterior ou de trânsito para outro.

Falar do ecomosaico usando estreitamente índices é tomar a parte pelo todo, e então a metáfora do ecomosaico resulta numa sinédoque. Não existe medida de informação única, nem sequer o conceito de informação é o mesmo em todo o lado. No caso mais frequente, informação é algo que diminui a incerteza. Para Rosnay (1995), a informação é tempo virtual. Noutro lugar, defende-se que o processo de valorização numérica de um habitat envolve um número complexo, equivalente a um vetor real bidimensional, e apresenta-se outro índice de informação baseado num procedimento distinto: uma medida de variabilidade de um sistema de variáveis de Bernoulli características do mosaico (CASQUILHO, 2009).

\section{CONCLUSÕES}

O conjunto de índices de informação que se apresentou neste trabalho, representados na sua relação funcional genérica $I_{w}(\alpha ; x)=U_{\alpha}(y \mid w)$, representa um exercício de abdução: trata-se de uma generalização da função da entropia estatística de Shannon, de forma a dar conta da internalização de valores característicos dos habitats numa medida de diversidade da paisagem, depois estendida a índices mais abrangentes. Pode servir para calibrar medidas de impacto de mudanças na paisagem nesta ou naquela perspectiva, consoante a natureza do conjunto de valores característicos utilizados.

No caso exemplificado, um esboço prospectivo de valorização dos habitats florestais na região de Nisa, em Portugal, concluiu-se, em face do procedimento adoptado, que se pode balizar uma solução de composição em que o montado de sobro e o pinhal manso alcançam valores percentuais de cerca de $30 \%$ a $40 \%$ ou, mesmo, $50 \%$ no caso do sobro, enquanto os outros habitats considerados, eucaliptal, pinhal bravo e azinhal, tomam posições significativamente inferiores, que rondam entre menos de 5\% e cerca de 15\%. Essa solução é uma referência e nada mais do que isso, pois supõe a homogeneidade biofísica da região e a ausência de restrições.
Falou-se aqui de abdução no seu sentido lato, como desenvolveu Peirce: a abdução é o processo de formar uma hipótese explicativa, sugerindo que alguma coisa pode ser operativa (MOURÃO, 2007). No mesmo âmbito, Batens (2007) referiu que a visão contemporânea da racionalidade científica é a de que a ciência é orientada pelo conteúdo e que o caminho específico seguido para resolver um problema é determinado por escolhas que são permitidas dentro do procedimento.

\section{AGRADECIMENTOS}

Ao engenheiro Carlos Janeiro, pelo documento citado (PMDFCI, 2007) e pelos esclarecimentos.

\section{REFERÊNCIAS}

ALLEN, B.; KON, M.; BAR-YAM, Y. A new phylogenetic diversity measure generalizing the shannon index and its application to phyllostomid bats. The American Naturalist, v.174, p.236-243, 2009.

ALLEN, C. R.; GUNDERSON, L. H.; HOLLING, C. S. Commentary on part one articles. In: GUNDERSON, L. H.; ALLEN, C. R.; HOLLING, C. S. (Eds.) Foundations of ecological resilience. Washington: Island Press, 2010a. p.3-18.

ALLEN, C. R.; GUNDERSON, L. H.; HOLLING, C. S. Commentary on part three articles. In: GUNDERSON, L. H.; ALLEN, C. R.; HOLLING, C. S. (Eds.) Foundations of ecological resilience. Washington: Island Press, 2010b. p.301-307.

ALVES, A. A. M. O conceito florestal de uso múltiplo sob uma óptica de desenvolvimento económico. Agros, v.46, n.2, p.141-150, 1963.

AMARAL, M. V. et al. Dinâmica da cobertura vegetal (1980-2004) em um projeto de produção florestal, nos municípios de Bugre e Ibapa, MG. Revista Árvore, v.33, n.3, p.515-325, 2009.

BATENS, D. Content guidance in formal problem solving processes. In: POMBO, O.; GERNER, A. (Eds.) Abduction and the process of scientific discovery. Lisboa: Centro de Filosofia das Ciências da Universidade de Lisboa, 2007. p.121-156.

Revista Árvore, Viçosa-MG, v.36, n.2, p.321-329, 2012 
BLACKWELL, D.; GIRSHICK, M. A. Theory of games and statistical decisions. New York: Dover Publications, 1979. 355p.

BOWMAN, D. Using landscape ecology to make sense of Australia's last frontier. In: WU, J.; HOBBS, R. (Eds.) Key topics in landscape ecology. Cambridge: Cambridge University Press, 2007. p.214-226.

CANN, R.; KEMPSON, R.; GREGOROMICHELAKI, E. Semantics - an introduction to meaning in language. New York: Cambridge University Press, 2009. 292p.

CASQUILHO, J.; NEVES, M.; REGO, F. Extensões da função de Shannon e equilíbrio de proporções - uma aplicação ao mosaico de paisagem. Anais do Instituto Superior Agronomia, v.46, p.77-99, 1997.

CASQUILHO, J.P. Complex number valuation of habitats and information index of the landscape mosaic. Silva Lusitana, v.17, n.2, p.171-180, 2009.

CAUQUELIN, A. A invenção da paisagem. Lisboa: Edições 70, 2008. 151p.

CEMIN, G.; PERICO, E.; REMPEL, C. Composição e Configuração da Paisagem da Sub-bacia do Arroio Jacaré, Vale do Taquari, RS, com Ênfase nas Áreas de Florestas. Revista Árvore, v.33, n.4, p.705-711, 2009.

FORMAN, R. T. T. The pine barrens of New Jersey: an ecological mosaic. In: FORMAN, R. T.T (Ed.) Pine barrens: ecosystem and landscape. New York: Academic Press, 1979. p.569-585.

FORMAN, R. T. T.; GODRON, M. Patches and structural components for a landscape ecology. Bioscience, v.31, p.733-740, 1981.

FORMAN, R. T. T. Ecologically sustainable landscapes: the role of spatial configuration. In: ZONNEVELD, I. S.; FORMAN, R. T. T. (Eds.). Changing landscapes: an ecological perspective. London: Springer-Verlag, 1990. p.261-278.
FORMAN, R. T. T. Land mosaics - the ecology of landscapes and regions. Cambridge: Cambridge University Press, 1995. 632p.

GASPAR, J.; FIDALGO, B. Evolução do uso do solo e avaliação do valor paisagístico e de recreio na área de paisagem protegida da Serra do Açor. Silva Lusitana, v.10, n.2, p.179-194, 2002.

GILBOA, I. Theory of decision under uncertainty. New York: Cambridge University Press, 2009. 214p.

GILLER, P. S. Community structure and the Niche. London: Chapman and Hall, 1984. 176p.

GOMES, A. M. A. Uma alternativa sectorial. Lisboa: Publicações Ciência e Vida, 1985. 142p.

HANSEN, V. L. Geometry in nature, Wellesley: A K Peters, 1993. 238p.

HOF, J.; FLATHER, C. Optimization of landscape pattern. In: WU, J.; HOBBS. R. (Eds.) Key topics in landscape ecology. Cambridge: Cambridge University Press, 2007. p.143-160.

HOLLING, C. S. Resilience and stability of ecological systems. Annual Review of Ecology and Systematics, v.4, p.1-23, 1973.

KLIR, G. J. Uncertainty and information - foundations of generalized information theory. New Jersey: John Wiley \& Sons, 2006. 499p.

KORNREICH, P. Mathematical Models of Information and Stochastic Systems. Boca Raton: CRC Press, 2008. 364p.

LEPECKI, M.L. A mãe promíscua: sobre natureza e paisagem. Finisterra, v. 36, n.72, p:141-147, 2001.

LI, H.; WU, J. Landscape pattern analysis: key issues and challenges. In: WU, J.; HOBBS, R. (Eds.) Key topics in landscape ecology. Cambridge: Cambridge University Press, 2007. p.39-61. 
MOREIRA, F.; REGO, F. C.; FERREIRA, P. G. Temporal (1958-1995) pattern of change in a cultural landscape of northwestern Portugal: implications for fire occurrence. Landscape Ecology, v.16, n.6, p.557-567, 2001.

MOURÃO, J. A. Abduction and metaphor. Tropism of truth. In: POMBO, O.; GERNER, A. (Eds.). Abduction and the process of scientific discovery. Lisboa: Centro de Filosofia das Ciências da Universidade de Lisboa, 2007. p.265-282.

NAGENDRA, H.; GADGIL, M. Biodiversity assessment at multiple scales: Linking remotely sensed data with field information.

Proceedings of the National Academy of Sciences of the United States of America, v.96, n.16, p.9154-9158, 1999.

PARIKH, P.; CLARK, R. An introduction to equilibrium semantics for natural language. In: PIETARINEN, A. H. (Ed.) Game theory and linguistic meaning. Oxford: Elsevier, 2007. p.149-158.

PETITOT, J. Morphologie et esthétique. Paris: Maisonneuve et Larose, 2004. 375p.

PMDFCI. Plano Municipal de Defesa da Floresta Contra Incêndio de Nisa. Câmara Municipal de Nisa e Florasul, 2007. 327p.

RICOTTA, C. Bridging the gap between ecological diversity indices and measures of biodiversity with Shannon's entropy: comment to Izsák and Papp. Ecological Modelling, v.152, n.1, p.1-3, 2002.
ROSNAY, J. L’Homme Symbiotique regards sur le troisième millénaire. Paris: Éditions du Seuil, 1995. 350p.

SANTOS, J. M.L. Valuing alternative bundles of landscape attributes: cost-benefit analysis for the selection of optimal landscapes. Finisterra, v.36, n.72, p.207-239, 2001.

SHANNON, C. E. A mathematical theory of communication. Bell System Technical Journal, v.27, p.379-423, 1948.

SIMPSON, E. H. Measurement of diversity. Nature, v.163, p.688, 1949.

SOLÉ, R. V.; BASCOMPTE, J. Selforganization in complex ecosystems. Princeton: Princeton University Press, 2006. 373p.

STRATTON-LAKE, P. Kant, duty, and moral worth. New York: Barnes \& Noble, 2004. 168p.

WEIRICH, P. Decision space: multidimensional utility analysis. Cambridge: Cambridge University Press, 2001. 272p.

WU, J.; HOBBS, R. Landscape ecology: the state-of-the-science. In: WU, J.; HOBBS, R. (Eds.) Key topics in landscape ecology. Cambridge: Cambridge University Press, 2007. p. 271-287.

ZONNEVELD, I. Scope and concepts of landscape ecology as an emerging science. In: ZONNEVELD, I.; FORMAN, R. T. T (Eds.). Changing landscapes: an ecological perspective. New York: Springer-Verlag, 1990. p.1-20. 
